\title{
DEVELOPMENT OF DESIGN SOLUTIONS FOR ELECTRIC DELIVERY VEHICLES WITH A GVM OF 4.25 T
}

\author{
Łukasz GoŁęBiewski, Piotr Żach \\ Warsaw University of Technology, Institute of Machine Design Fundamentals, Warsaw, Poland \\ e-mail: lukasz.golebiewski.dokt@pw.edu.pl; piotr.zach@pw.edu.pl
}

\begin{abstract}
Van bodies are based on frame structures produced of aluminum alloy profiles. Searching for new directions in the development of vehicle bodies for newly designed eVans led to becoming focused on currently unused polymer composites in this area. The authors of the article designed a composite body for a delivery vehicle. They conducted detailed, numerical comparative analyses of the commercial body and the developed solution. The obtained results clearly confirmed the correctness of the formulated thesis about the possibility of designing bodies dedicated to electric vehicles made of structural composites.
\end{abstract}

Keywords: transport body, delivery vehicle, eVan, composite materials, FEM analysis

\section{Introduction}

Nowadays, van bodies are based on frame structures produced of aluminum alloy profiles (Szabo, 2018). Searching for new possibilities in the adaptation of vehicle body construction solutions in newly designed eVans (Lubczyński and Zuska, 1998) led to focusing on currently unused materials - polymer composites (Beer et al., 2014). The authors assessed the possibility of using fibre composites to produce an open body for a delivery vehicle.

The aim of the study was to assess stiffness and strength properties and to determine tension of the structure taking into account operational loads of the vehicle. The analysis took into account the ways of loading and the requirements of vehicle qualifications resulting from the approval regulations for delivery vehicles (Directive 2007/46/EC).

\section{Characteristics of the construction of a body for a delivery vehicle}

Using the Finite Element Method, an analytical verification of an open body frame for a delivery vehicle up to $3.5 \mathrm{t}$ was carried out. The frame was made of AW-6060 aluminum alloy developed by ALU-S.V. (2018). The construction of the body was designed to be adapted on a long wheelbase chassis cab. Based on (New Fiat Ducato, 2016; Reimpell and Betzler, 2001; Sobczak and Kubat, 2017; Zieliński, 2016), the following assumptions were made:

- vehicle chassis weight (without the body) $m_{p}=1780 \mathrm{~kg}$ - in accordance with the EC certificate of conformity No. e9*2007/46*6109*00

- $m_{p}$ - vehicle weight (chassis to be developed) - unladen weight (with all fluids, fuel tank $90 \%$ full), $m_{p k}=1860 \mathrm{~kg}$ - in accordance with the EC certificate of conformity No. e $9 * 2007 / 46 * 6109 * 00$

- $l_{c}$ - total length of the vehicle $=5943 \mathrm{~mm}$

- $s_{c}$ - total width of the vehicle $=2050 \mathrm{~mm}$

- $l_{z}$ - body length $=3505 \mathrm{~mm}$

- $s_{z}$ - body width $=2350 \mathrm{~mm}$ 
- $b$ - vehicle track width, $b=1790 \mathrm{~mm}$

- $l_{o}$ - wheelbase, $l_{o}=4035 \mathrm{~mm}$

- $h_{p}$ - height of the centre of mass of the vehicle (chassis to be developed), $h_{p}=642 \pm 3 \mathrm{~mm}$

- $m_{z}$ - body mass, $m_{z}=1910 \mathrm{~kg}$

- $h_{r}$ - height of the upper surface of the construction frame, $h_{r}=642 \pm 3 \mathrm{~mm}$

- $m_{c \max }$ - maximum allowable total load, $m_{c \max }=3345-3500 \mathrm{~kg}$

- $m_{p \max }-$ maximum allowable load on the front axle, $m_{p \max }=1850 \mathrm{~kg}$

- $m_{t \max }$ - maximum allowable load on the rear axle, $m_{t \max }=2000 \mathrm{~kg}$

- $n_{u}$ - usable load capacity with the driver, $n_{u}=1640 \mathrm{~kg}$

Characteristic data of the structure:

$>$ support frame:

- $l_{z r}$ - frame length $=3505 \mathrm{~mm}$

- $s_{z r}$ - frame width $=2280 \mathrm{~mm}$

- $w_{z r}$ - frame height $=202 \mathrm{~mm}$

Two different versions of the body development were considered, taking into account I-beams with a height of $130 \mathrm{~mm}$ (T 130) and $108 \mathrm{~mm}$ (T 108):

- $h_{1}$ - height of the centre of mass of the aluminum frame assembly ( $Z$ coordinate) with a T 130 beam, $h_{1}=799 \mathrm{~mm}$

- $h_{2}$ - height of the centre of mass of the aluminum frame assembly ( $Z$ coordinate) with a T 108 beam, $h_{2}=771 \mathrm{~mm}$

- $h_{r 1}$ - height of the upper surface of the frame, assembly with a T 130 beam, $h_{r 1}=860 \mathrm{~mm}$

- $h_{r 2}$ - height of the upper surface of the frame, assembly with a T 108 beam, $h_{r 2}=838 \mathrm{~mm}$

- $m_{r 1}$ - frame weight assembly with a T 130 beam, $m_{r 1}=101 \mathrm{~kg}$

- $m_{r 2}$ - frame weight assembly with a T 108 beam, $m_{r 2}=99 \mathrm{~kg}$

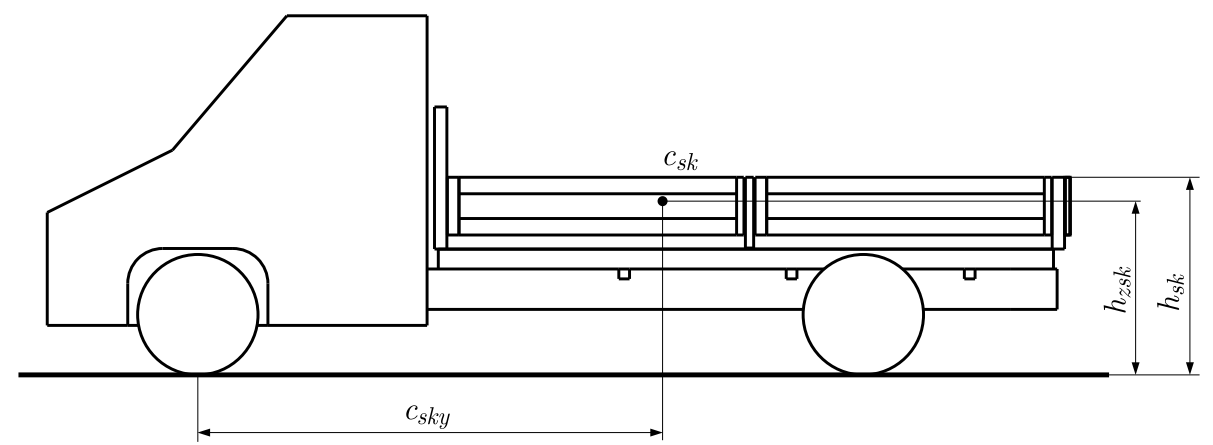

Fig. 1. Location of the characteristic points of the vehicle with the support frame of the body

- open body - assumptions for the assessment of the location of characteristic points in the structure are presented in Fig. 1:

- $l_{z s k}$ - open body length $=3560 \mathrm{~mm}$

- $s_{z s k}$ - open body width $=2350 \mathrm{~mm}$

- $w_{z s k}$ - open body height $=400 \mathrm{~mm}$

- $h_{z s k}$ - height of the centre of mass of the open body, $h_{z s k}=1063 \mathrm{~mm}$

- $c_{s k x}$ - position of the centre of mass of the open body assembly with a T108 beam in relation to the transverse axle of the vehicle ( $X$ coordinate), $c_{r x 1}=0 \mathrm{~mm}$

- $c_{s k y}$ - position of the centre of mass of the open body assembly with a T108 beam in relation to the longitudinal axle of the vehicle ( $Y$ coordinate), $c_{r y 1}=2746 \mathrm{~mm}$

- $h_{s k 1}$ - height of the upper edge of the open body, $h_{s k}=1238 \mathrm{~mm}$

- $m_{s k}$ - weight of the open body, $m_{s k}=72.8 \mathrm{~kg}$ 


\section{Analysis of the frame and open platform with AlMgSi 0.7}

A number of load cases to which the structure may be subjected during operation were tested, i.e.:

- a load originating from: cargo evenly distributed over the entire surface of the floor panel on the surface of a Euro-pallet with dimensions of $1200 \mathrm{~mm} \times 800 \mathrm{~mm}$ placed in different places of the open platform, assuming that the sum of loads was equal to the usable load capacity of the vehicle for the type under consideration,

- loads transmitted through the frame of the supporting structure for the installation of a tarpaulin on the open platform, coming from: the weight of the body frame for the installation of the tarpaulin, the weight of the tarpaulin lowered and secured and folded on one side, the centrifugal force of inertia of the vehicle motion along the curve with the maximum speed that guarantees safe operation of the vehicle, wind pressure: in the direction consistent with and against the action of the centrifugal force to the rear of the vehicle and a combination of variants.

The following load cases were formulated using (Editorial Board of the Automotive Engineering Manual, 2001; Lee et al., 2016; Reimpell and Betzler, 2001; Reński, 2004; Wang et al., 2019):

1) body dry weight, tarpaulin weight, wind pressure of $1300 \mathrm{~N}$ from the rear of the vehicle,

2) body dry weight, tarpaulin weight and crosswise centrifugal acceleration of inertia of $38 \mathrm{~m} / \mathrm{s}^{2}$

3) body dry weight, tarpaulin weight and wind pressure on the side surface of the body, with a total value of $2880 \mathrm{~N}$

4) body dry weight, tarpaulin weight, wind pressure on the side surface of the body with a total value of $2880 \mathrm{~N}$, crosswise centrifugal acceleration of inertia of $38 \mathrm{~m} / \mathrm{s}^{2}$ in the direction of wind pressure,

5) body dry weight, tarpaulin weight, the force of wind pressure on the side surface of the body with a total value of $2880 \mathrm{~N}$, crosswise centrifugal acceleration of inertia of $38 \mathrm{~m} / \mathrm{s}^{2}$ in the direction opposite to wind pressure,

6) body dry weight, tarpaulin weight, wind pressure on the side surface of the body with a total value of $2880 \mathrm{~N}$, crosswise centrifugal acceleration of inertia of $38 \mathrm{~m} / \mathrm{s}^{2}$ in the direction of wind pressure, wind pressure from the rear of the vehicle with a value of $1300 \mathrm{~N}$,

7) body dry weight, tarpaulin weight and wind pressure on the side surface of the body, with a total value of $2,880 \mathrm{~N}$.

The analyses were performed in SolidWorks (SolidWorks manual, 2019) with the use of beam and sheathing elements, in accordance with the conclusions of the publications (Aghav and Walame, 2016; Zuo et al., 2016). Boundary conditions were formulated in the points of fixing the body frame to the vehicle frame. At the points marked in Fig. 2, displacements in $X$, $Y$ and $Z$ directions were fixed. A number of variants were considered.

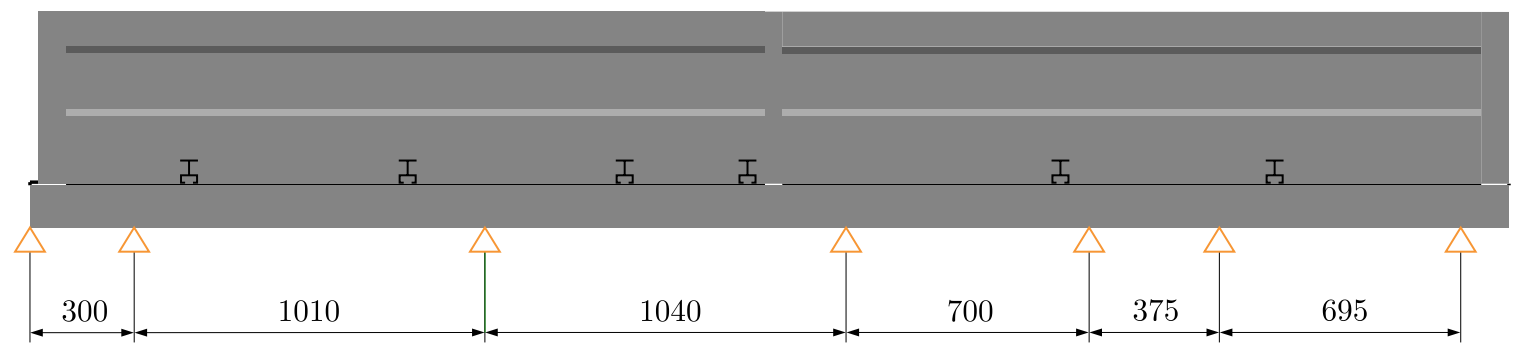

Fig. 2. Diagram of boundary conditions adopted during the analyses 
The classic beam solution of the body structure (Zhang et al., 2020) was compared with the original design based on grating plates. Figures 3 and 4 show selected results of numerical analyses, including the case of the body load: in the form of a pallet with a weight of $14.5 \mathrm{kN}$, the dry weight of the body frame for the installation of the tarpaulin, lowered and secured, the centrifugal force of inertia and the pressure force of the side wind with a direction consistent with the force of inertia.

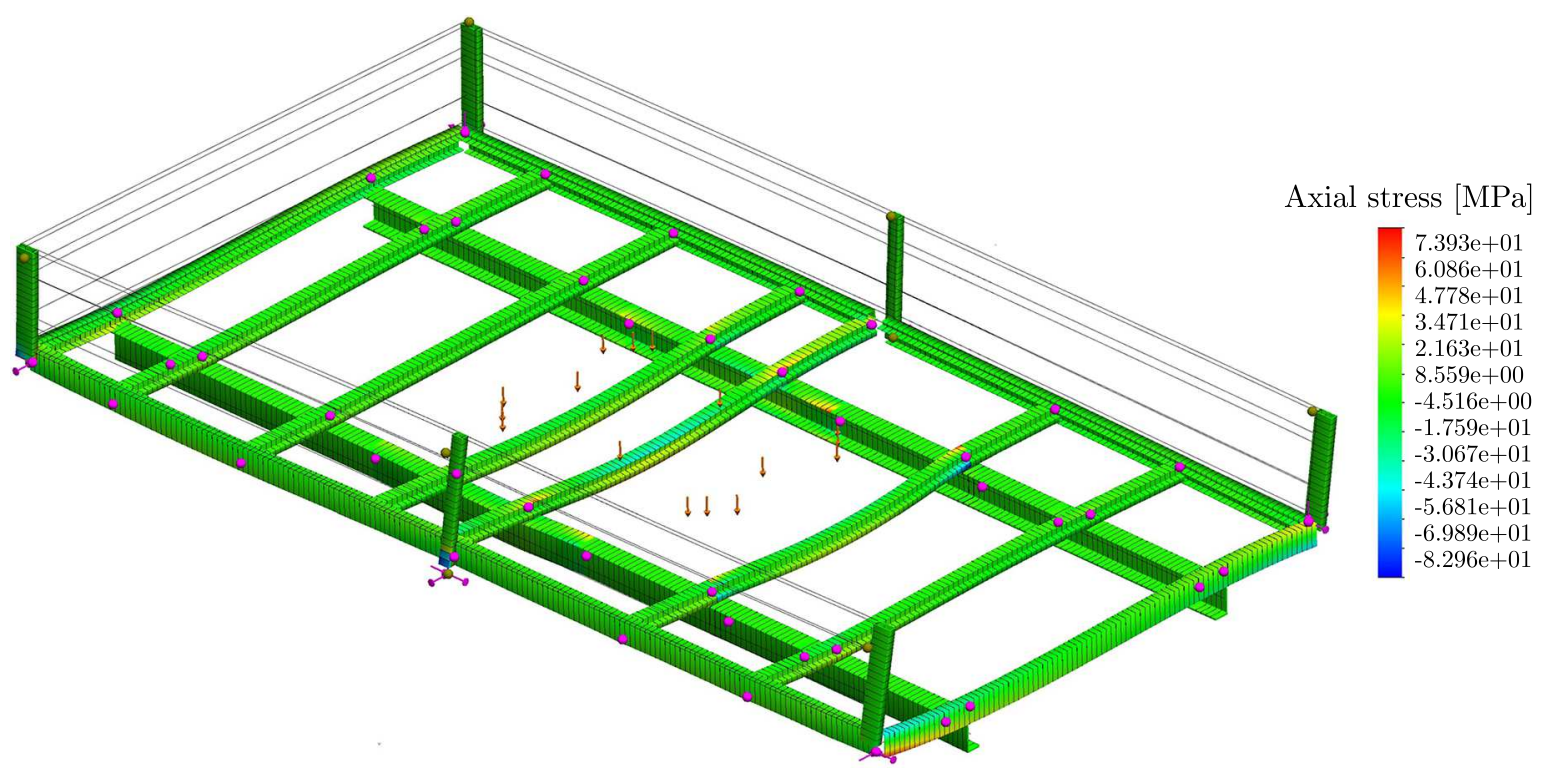

Fig. 3. Map of axial stresses in the body structure beams under a load in the form of a pallet weighing $14.5 \mathrm{kN}$, dry weight of the body frame for the installation of the tarpaulin, lowered and secured, the centrifugal force of inertia and the pressure force of the side wind with a direction consistent with the force of inertia

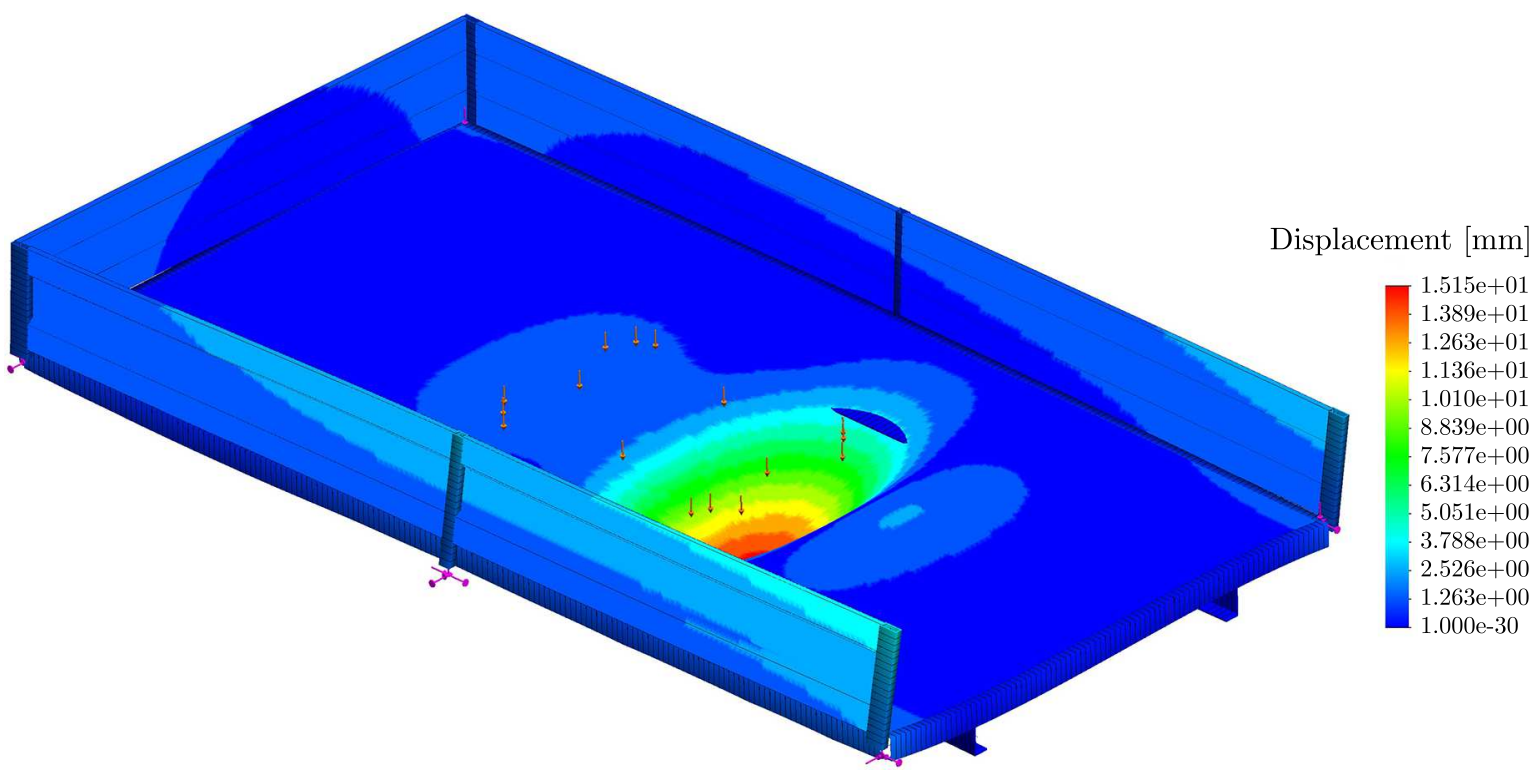

Fig. 4. Map of displacements in the body structure under a load in the form of a pallet weighing $14.5 \mathrm{kN}$, dry weight of the body frame for the installation of the tarpaulin, lowered and secured, the centrifugal force of inertia and the pressure force of the side wind with a direction consistent with the force of inertia with a value of $4800 \mathrm{~N}$ 


\section{Analysis of the frame and open platform made of composite materials}

Based on the experience gained, a composite, polyester and glass body for a delivery vehicle was designed and assessed for its behaviour and compliance with the guidelines (Directive $2007 / 46 / \mathrm{EC})$.

The design solution of the original composite vehicle body is based on the use of commercially available composite grating plates that enable one to obtain a homogeneous structure of the cargo area floor plate. Longitudinal beams (side members) were attached directly to the vehicle support frame. The floor plate was placed on composite longitudinal beams. The height of the beams was the subject of reflections due to the necessity to ensure clearance in the rear suspension system of the vehicle, the location of the centre of gravity of the body and stability, in particular in a curved motion. The floor plate is framed with peripheral profiles to which integral elements of the body - elements of the support structure of the tarpaulin covering the cargo and sides are attached. The designed solution is dedicated to electric delivery vehicles, the so-called eVans (Zhang et al., 2020; Wang, 2012; Żebrowski et al., 2018). The model of the designed composite body is shown in Fig. 5, the profiles used are shown in Figs. 6-8, and the composite support grating panel in Fig. 9.
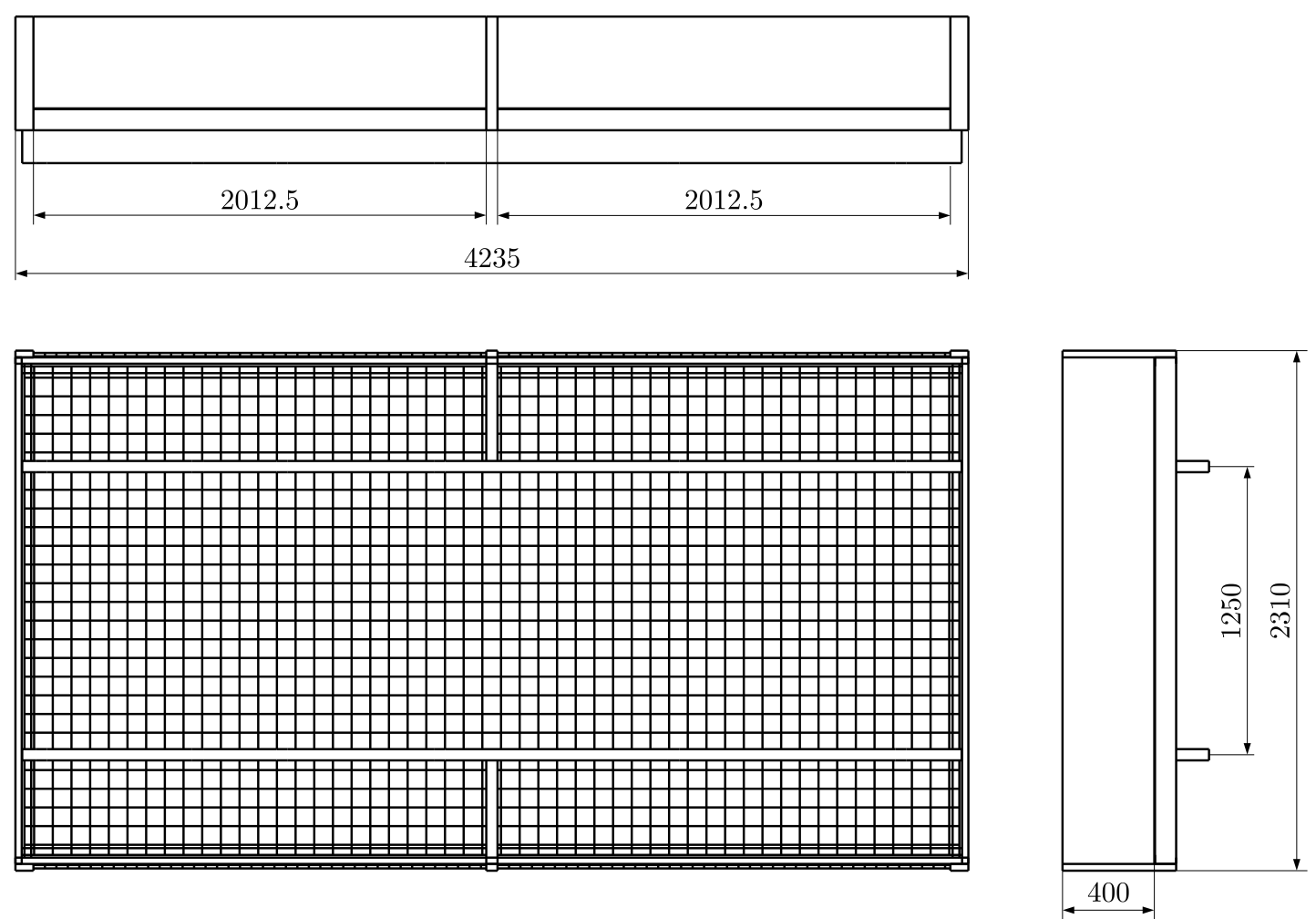

Fig. 5. Structural model of the open body designed on the basis of a grating plate

(a)

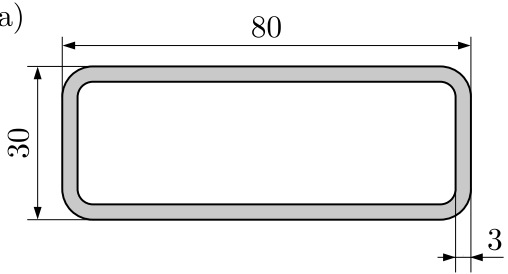

(b)

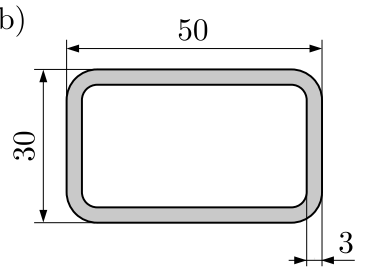

Fig. 6. Cross-sections of supporting pillars of the open body: (a) corner, (b) middle 


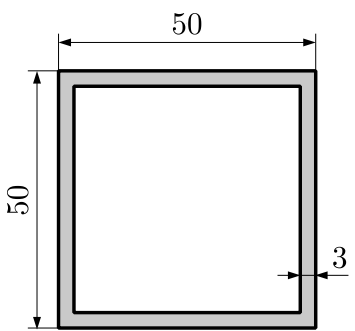

Fig. 7. Cross-section of a beam that strengthens the fastening of the central pillar

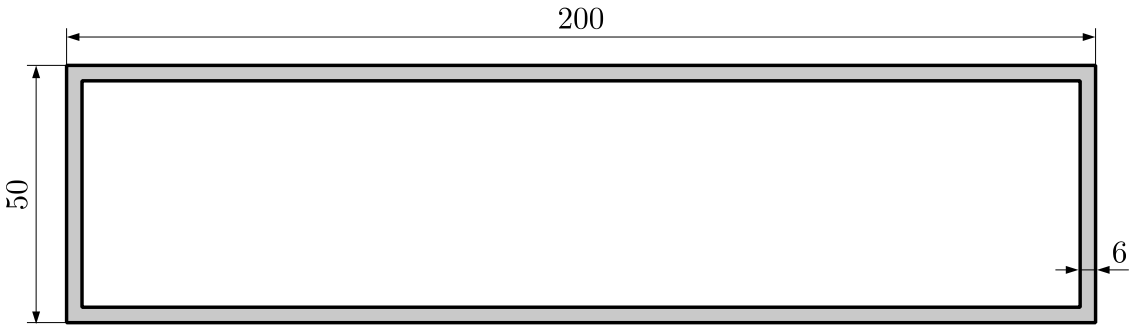

Fig. 8. Cross-section of the longitudinal beam
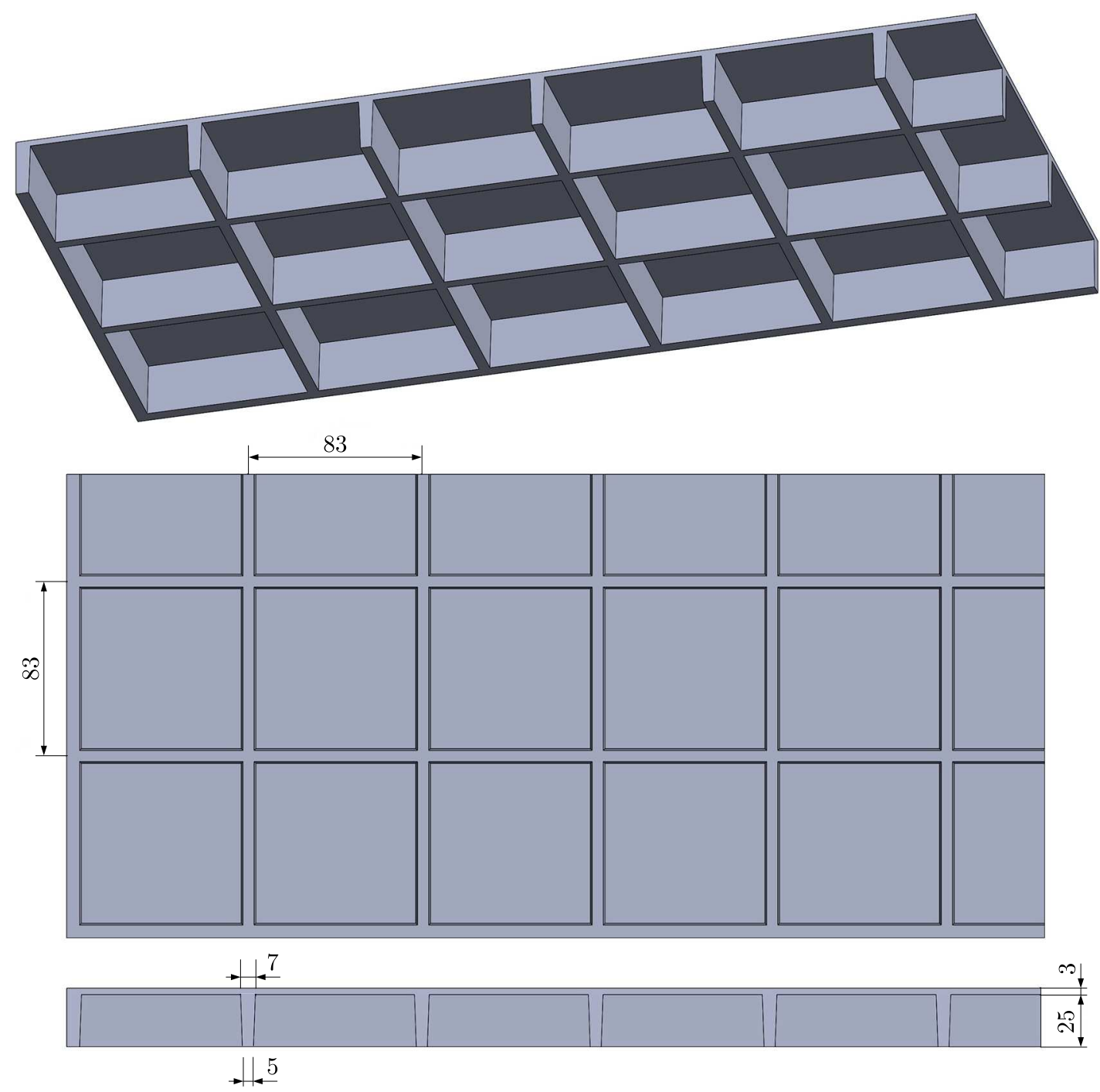

Fig. 9. Composite support grating plate of the body 
Figures 10 and 11 show the nature of the work of the composite support frame with the open body for the same load variant as in the case of the aluminum alloy body.

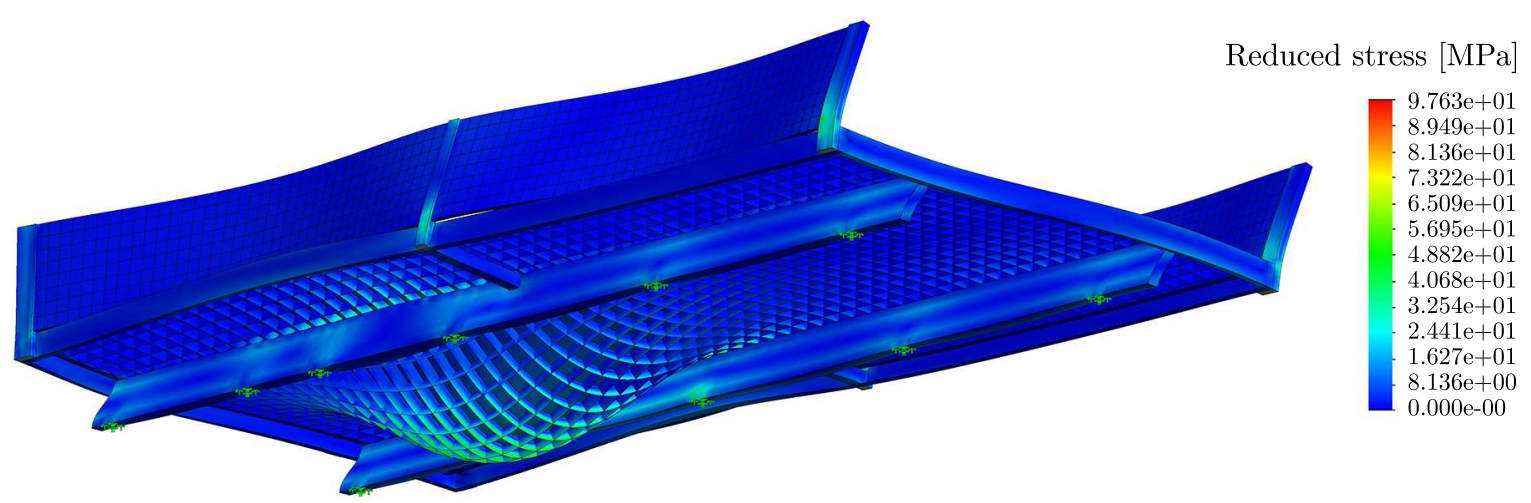

Fig. 10. Map of reduced stresses in the structure of the composite open body under a load in the form of a pallet with a weight of $14 \mathrm{kN}$, dry weight of the structure for the installation of the tarpaulin, centrifugal force of inertia and the pressure force of the side wind with a direction consistent with the force of inertia of $4800 \mathrm{~N}$

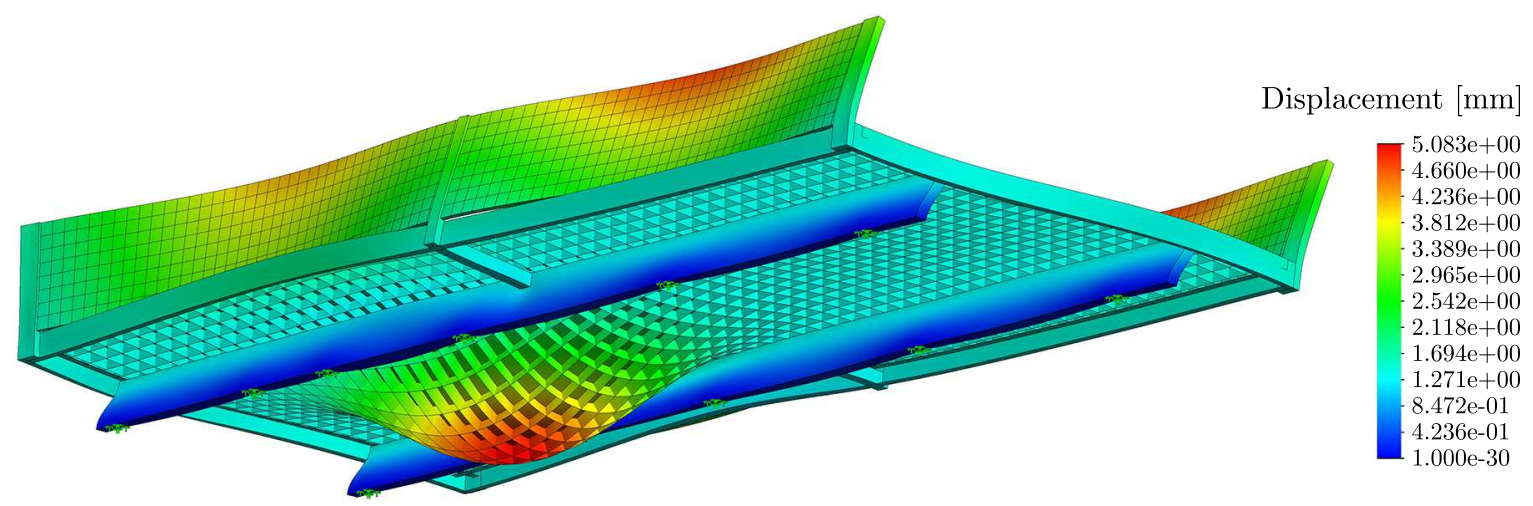

Fig. 11. Map of displacements of the composite structure of the open body under a load in the form of a pallet with a weight of $14.5 \mathrm{kN}$, dry weight of the structure for the installation of the tarpaulin, centrifugal force of inertia and the pressure force of the side wind with a direction consistent with the force of inertia of $4800 \mathrm{~N}$

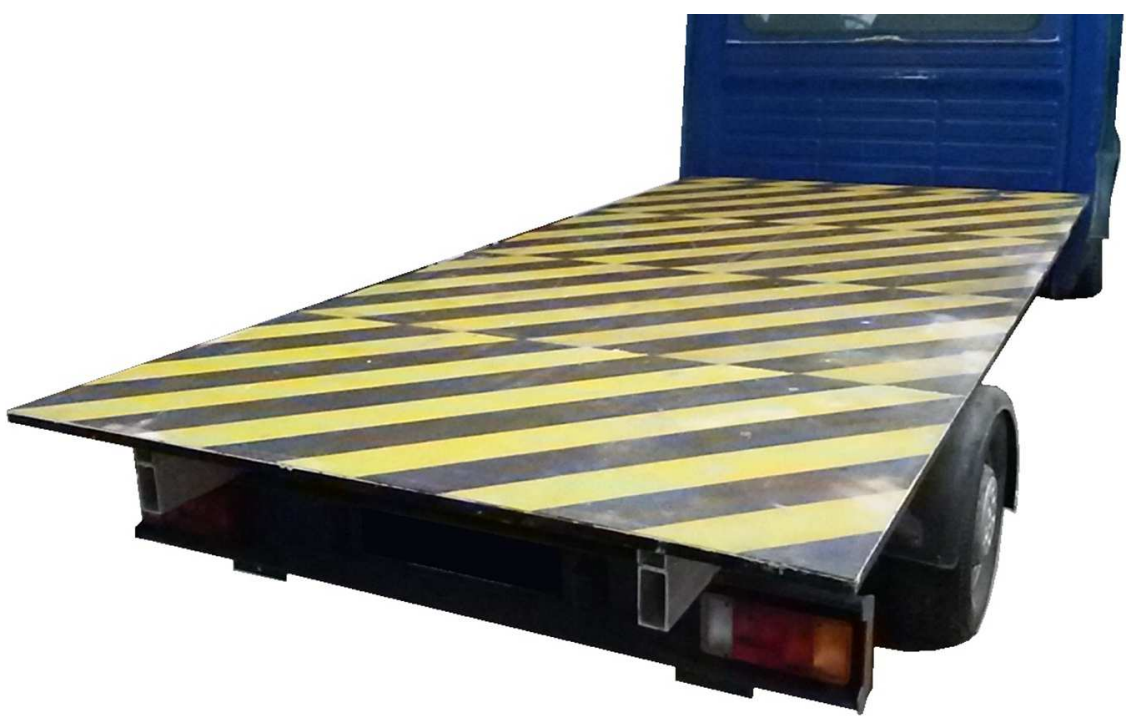

Fig. 12. Photo of a demonstrator of the body composite floor plate stand during assembly 
A composite floor plate stand was made for experimental tests (Fig. 12). Obtaining convergence between the experimental and numerical results will be the basis for the implementation of a prototype composite body dedicated to electric delivery vehicles.

\section{Summary}

The subject of the article was the stiffness and strength analysis of the open body frame made of AlMgSi 0.7 for a delivery vehicle in the chassis cab with a platform variant. The solution designed by the manufacturer ALU-SV (ALU-S.V., 2018) was verified on the basis of the physical measurements of the object.

The stability coefficient of the body frame for the installation of the tarpaulin was determined, and the loads transferred by the system to the structural elements of the open platform and the support frame of the body were determined. The phenomena occurring in the structure subjected to static loads with the structure dry weight, tarpaulin weight, external operational loads and variants of the above-mentioned impacts were verified according to information in publications (Aghav and Walame, 2016; Zuo et al., 2016).

It was found that for most cases the main factor influencing the level of maximum stresses in the support frame and body is the weight of the transported cargo. In the second variant, the load was determined by wind pressure on the side surface of the body, which is in line with the conclusions of the publication (Wang, 2012; Lew et al., 2011).

The difference in masses of the analyzed variants of bodies with AlMgSi 0.7 and polyester composite amounts to $41 \mathrm{~kg}$, which is $20 \%$ of the total mass made of aluminum alloy.

The strength of the structure for both verified solutions is similar. The differences are within the range of [\%]. The estimated stiffness in the case of the composite body is $(x)$. The designed solution is characterized by a high safety factor (alu-composite relation). Stress and displacement levels and the nature of the structure work for the verified construction materials are comparable.

It was found that there is a strong dependence between the location of cargo and the nature of the body work made of AlMgSi 0.7. In the case of the solution using composite gratings, the local influence of cargo placement was limited, which made it possible to obtain a uniform tension of the entire structure of the body. In the original development solution, the number of components was reduced, which will translate into a reduced cost of the structure and production time.

The direction of using composite materials in vehicle structures opens up new possibilities in the design and adaptation of delivery vehicles, especially electric ones. The demonstrated possibility of reducing weight of the structure through the use of composite materials allows one to increase load capacity or compensate for the increase in weight resulting from the use of electric drive systems. For comparable vehicle operating conditions, it will be possible to reduce energy consumption per unit of distance.

\section{References}

1. Aghav H.L., Walame M.V., 2016, Stress analysis and fatigue analysis of front axle of heavy-duty truck using ANSYS Ncode design life for different loading cases, International Journal of Engineering Research and Applications, 6, 6, 78-82.2

2. ALU-S.V., 2018, Box Bodies, Commercial Materials

3. Beer F., DeWolf J., Johnston E.R. JR, Mazurek D., 2014, Mechanics of Materials, New York: McGraw-Hill Education 
4. Directive 2007/46/EC of the European Parliament and of the Council of 5 September 2007 establishing a framework for the approval of motor vehicles and their trailers, and of systems, components and separate technical units intended for such vehicles (Framework Directive)

5. Editorial Board of the Automotive Engineering Manual, 2001, Automotive Engineering Manual (for Design) (in Chinese), Beijing, China, China Communications Press

6. Lee E.-M., Shim D.-S., Son J.-Y., Baek G.-Y., Yoon H.-S., Ro K.-B., 2016, Study on design of progressive dies for manufacture of automobile structural member using DP980 advanced high strength steel, Journal of Mechanical Science and Technology, 30, 2, 853-864

7. Lew H.S., Bao Y., Sadek F., Main J.A., Pujol S., Sozen M., 2011, An experimental and computational study of reinforced concrete assemblies under a column removal scenario, NIST Technical Note, $\mathbf{1 7 2 0}$

8. Lubczyński M., ZuskA A., 1998, Analysis of use of utility vans, Czasopismo Techniczne. Mechanika, 95, 6, 276-283

9. New Fiat Ducato 2016, Converters' and Upfitters' Manual

10. Reimpell J., Betzler W.J., 2001, Car Chassis. Basics of Construction (in Polish), Wydawnictwa Komunikacji i Łączności WKE

11. Reński A., 2004, Automobile Construction: Braking and Steering Systems and Suspension (in Polish), Publishing House of the Warsaw University of Technology

12. Sobczak P., Kubat W., 2017, Analysis of changes in the structure of motor vehicles in relation to sustainable European Union transport policy plans as an element of transport management, Autobusy, 6

13. SolidWorks Manual, 2019

14. Szabo F.J., 2018, Optimization of springs applied in vehicle suspension structure, [In:] Vehicle and Automotive Engineering, K. Jármai, B. Bolló (Eds.), Cham, Springer, 585-596

15. WAng S., 2012, Finite Element Analysis and Structural Optimization of a Commercial Bus Frame (in Chinese), Master's Thesis, Wuhan University of Science and Technology, Wuhan, China

16. Wang X.C., Lu Q., Li R., Xu X., 2019, Numerical analysis on aerodynamic performance of different automobile body shape, IOP Conference Series: Earth and Environmental Science, 242, 3, 032027

17. Zhang H., Huang G., Yu D., 2020, Numerical modeling for the frame structure of light van-type electric truck, Science Progress, 103, April

18. Zieliński A., 2016, Construction of Car Bodies of Passenger Cars and Derived (in Polish), Wydawnictwa Komunikacji i Łączności WKŁ

19. Zuo W., Yu J., SAitou K., 2016, Stress sensitivity analysis and optimization of automobile body frame consisting of rectangular tubes, International Journal of Automotive Technology, 17, 5, 843-851

20. Żebrowski K., Detka T., MaŁek K., 2018, Comparative analysis of data from reports of CO2 emissions and total cost of ownership (TCO) of an electric vehicle in relations to a conventional vehicle, Maszyny Elektryczne - Zeszyty Problemowe, 119, 3 